\title{
Understanding jumping to conclusions in patients with persecutory delusions: working memory and intolerance of uncertainty
}

\author{
D. Freeman ${ }^{1 *}$, H. Startup ${ }^{1,2}$, G. Dunn ${ }^{3}$, E. Černis ${ }^{1}$, G. Wingham ${ }^{4}$, K. Pugh ${ }^{1}$, J. Cordwell ${ }^{4}$, H. Mander ${ }^{4}$ \\ and D. Kingdon ${ }^{4}$ \\ ${ }^{1}$ Department of Psychiatry, University of Oxford, Oxford, UK \\ ${ }^{2}$ Sussex Partnership NHS Trust, Worthing, UK \\ ${ }^{3}$ Centre for Biostatistics, Institute of Population Health, University of Manchester, Manchester, UK \\ ${ }^{4}$ Academic Department of Psychiatry, Faculty of Medicine, University of Southampton, Southampton, UK
}

\begin{abstract}
Background. Persecutory delusions are a key psychotic experience. A reasoning style known as 'jumping to conclusions' (JTC) - limited information gathering before reaching certainty in decision making - has been identified as a contributory factor in the occurrence of delusions. The cognitive processes that underpin JTC need to be determined in order to develop effective interventions for delusions. In the current study two alternative perspectives were tested: that JTC partially results from impairment in information-processing capabilities and that JTC is a motivated strategy to avoid uncertainty.
\end{abstract}

Method. A group of 123 patients with persistent persecutory delusions completed assessments of JTC (the 60:40 beads task), IQ, working memory, intolerance of uncertainty, and psychiatric symptoms. Patients showing JTC were compared with patients not showing JTC.

Results. A total of $30(24 \%)$ patients with delusions showed JTC. There were no differences between patients who did and did not jump to conclusions in overall psychopathology. Patients who jumped to conclusions had poorer working memory performance, lower IQ, lower intolerance of uncertainty and lower levels of worry. Working memory and worry independently predicted the presence of JTC.

Conclusions. Hasty decision making in patients with delusions may partly arise from difficulties in keeping information in mind. Interventions for JTC are likely to benefit from addressing working memory performance, while in vivo techniques for patients with delusions will benefit from limiting the demands on working memory. The study provides little evidence for a contribution to JTC from top-down motivational beliefs about uncertainty.

Received 30 October 2013; Revised 12 February 2014; Accepted 20 February 2014

Key words: Delusions, persecutory, reasoning, schizophrenia, working memory.

\section{Introduction}

Over the past decade there has been notable progress in understanding the cognitive mechanisms underlying a number of individual psychotic experiences. The greatest attention has been on delusional beliefs. A reasoning process repeatedly linked to delusions has been the presence of jumping to conclusions (JTC). This data-gathering bias has been found in 30 out of 38 clinical studies (see Garety \& Freeman, 1999, 2013). It is hypothesized that a limited data search leads to a rapid acceptance of implausible ideas,

\footnotetext{
* Address for correspondence: D. Freeman, Oxford Cognitive Approaches to Psychosis, University Department of Psychiatry, University of Oxford, Warneford Hospital, Oxford OX3 7JX, UK.

(Email: daniel.freeman@psych.ox.ac.uk)
}

without consideration of alternative explanations. Initial evidence indicates that JTC may be a predictor of delusion persistence (Menon et al. 2008; Dudley et al. 2013), and, importantly, that reducing the bias may reduce the delusion (Moritz et al. 2010; Waller et al. 2011). But what are the cognitive factors that contribute to the occurrence of JTC? In this study we test two hypotheses. First, JTC may result from information-processing limitations, as marked by working memory performance. Second, JTC may be a motivated strategy used to reduce difficulties coping with uncertainty.

JTC is assessed using 'the beads task', an experimental test originally based upon Bayesian probabilistic inference (Garety et al. 1991; Dudley et al. 1997; Warman et al. 2007; Lincoln et al. 2010). Participants are shown two jars varying in the proportion of differently 


\section{D. Freeman et al.}

coloured beads (e.g. one jar has 60 black beads and 40 yellow beads, the other jar has 40 black beads and 60 yellow beads). The jars are then hidden from view and the participant is told that the experimenter has selected one jar. The participant is asked to decide with certainty which jar has been chosen (e.g. the mainly black bead jar or the mainly yellow bead jar). The participant can request to see as many beads, drawn one by one from the selected jar, as he or she would like before making a decision. JTC is defined as deciding after being shown two or fewer beads. There is an easy version of the task, where the difference in the proportions of the two colours of bead is large (80:15), and a more difficult version of the task, where the two colours are present in proportions that are almost equal (60:40). About half of patients with delusions show JTC on the 80:15 beads task and about one-third on the 60:40 version (Garety \& Freeman, 2013).

Two recent large studies have tested the association of JTC with neuropsychological functioning. Although neuropsychological problems have been linked to the negative rather than the positive symptoms of psychosis (e.g. Ventura et al. 2009), there is a plausible case for expecting a contribution of working memory to the beads task -task instructions, two alternative possibilities, and the previous beads drawn have to be kept in mind. Garety et al. (2013) tested 126 patients with delusions, comparing working memory and premorbid intellectual functioning in those patients who jumped to conclusions with those who did not. There were no differences between the two groups in premorbid intelligence quotient (IQ) estimated with the Wechsler Test of Adult Reading (Wechsler, 2001). But on the 60:40 beads task in particular, there were associations of JTC with poorer working memory performance. The effect sizes were small to moderate. Falcone (2013) tested 112 patients with first-episode psychosis. IQ was assessed with five subtests of the working memory tasks from the Wechsler Adult Intelligence Scale III (WAIS-III; Wechsler, 1997). There were strong associations of JTC with IQ and working memory. The results of these two large studies are consistent with a number of earlier initial reports (e.g. Woodward et al. 2008), including a study indicating that data gathering may improve when memory load in the beads task is reduced (Menon et al. 2006). However, a study with 29 patients with early psychosis did not find a significant association of JTC with verbal working memory (Ormrod et al. 2012). Given the results of the largest studies, there are increasing grounds to believe that working memory limitations contribute to the presence of JTC.

An alternative perspective is that JTC is a motivated top-down strategy to forestall the presence of distressing uncertainty. Rather than dealing with doubt, and therefore potential confusion, a decision is rapidly made. A meta-cognitive belief-that uncertainty is to be avoided - could guide the data-gathering process when a decision is made. A potentially useful concept here is 'intolerance of uncertainty', developed to understand generalized anxiety disorder (Dugas et al. 1998). Intolerance of uncertainty is hypothesized to lead to magnification of problems in situations of little realistic risk, and worry is a person's misguided attempt to regain control over these magnified threats. The Intolerance of Uncertainty Scale (IUS) was developed to assess the concept (Freeston et al. 1994). Consistent with the idea that JTC is a motivated strategy to reduce the occurrence of distressing uncertainty, Broome et al. (2007) found higher levels of intolerance of uncertainty to be associated with less data gathering in the beads task in a combined high risk of psychosis $(n=35)$ and non-clinical control $(n=23)$ group.

Nonetheless, the relationship of data gathering with intolerance of uncertainly may not be straightforward. In their original scale development report, Freeston et al. (1994) speculated a potentially reverse effect: that intolerance of uncertainty may lead to 'high degrees of evidence before a decision can be made'. Furthermore, Dudley et al. (2011) found no association between JTC and intolerance of uncertainty in 77 patients recruited from a first-episode service. Similarly, in individuals with eating disorders, Sternheim et al. (2011) found no association of intolerance of uncertainty with performance on the beads task. It is also notable that a concept related to intolerance of uncertainty is 'need for closure', defined as 'individuals' desire for a firm answer to a question and an aversion toward ambiguity' (Kruglanski \& Webster, 1996). However, in a study with 187 patients with psychosis (Freeman et al. 2006), need for closure was unrelated to JTC. Although based upon similar concepts, the standard measures of intolerance of uncertainty and need for closure show only small to moderate associations (Berenbaum et al. 2008). Intolerance of uncertainty, and its relationship to JTC, remains to be tested in a large group of patients with current delusions.

The aim of the present study was to examine JTC in patients with delusions in relation to both neuropsychological functioning and intolerance of uncertainty. It was predicted that JTC would be associated with poorer working memory, lower IQ and greater intolerance of uncertainty. The association of JTC with working memory was expected to remain, even when controlling for levels of intellectual functioning. The association of JTC with intolerance of uncertainty was expected to remain, even when controlling for levels of worry. Hence information-processing constraints 
and top-down motivations were both expected to contribute to the occurrence of JTC.

\section{Method}

\section{Participants}

A total of 123 patients with persecutory delusions completed the JTC task during the baseline assessment (prior to randomization) of a clinical trial (ISRCTN23197625) (Freeman et al. 2012a). Patients with persecutory delusions were recruited from two mental health National Health Service (NHS) Trusts: Oxford Health NHS Foundation Trust and Southern Health NHS Foundation Trust. The inclusion criteria were: a current persecutory delusion as defined by Freeman \& Garety (2000); scoring at least 3 on the conviction scale of the Psychotic Symptom Rating Scales (PSYRATS)-delusions scale (i.e. at least $50 \%$ conviction in the delusion) (Haddock et al. 1999); that the delusion had persisted for at least 3 months; a diagnosis from the treating clinical team of 'non-affective psychosis', including schizophrenia, schizo-affective disorder and delusional disorder; a clinically significant level of worry, as indicated by scores above 44 on the Penn State Worry Questionnaire (PSWQ; Startup \& Erickson, 2006); aged between 18 and 65 years; and no changes to medication in the past month. Only eight patients with persecutory delusions were excluded from entering the trial because of an insufficient level of worry as assessed by the PSWQ. Criteria for exclusion were: a primary diagnosis of alcohol or substance dependency or personality disorder; organic syndrome or learning disability; a command of spoken English inadequate for engaging in therapy; and currently having individual cognitivebehavioural therapy.

\section{Assessments}

JTC: the beads task (Dudley et al. 1997; Garety et al. 2005)

Data gathering was assessed with a probabilistic reasoning task that has been extensively used with individuals with delusions. Participants are asked to request as many pieces of evidence (coloured beads) as they would like before making a decision (from which of two hidden jars the beads are drawn). The two jars have beads of two different colours in opposite ratios. The ratio of beads used in the current study was 60:40. The key variable is the number of beads requested before making a decision. Requesting two or fewer beads is classified as JTC. A categorical method of assessing JTC is used to capture the extreme form of the bias and because the number of draws to decision is not a normally distributed continuous scale (the informational value of each additional bead varies).

WAIS-III (Wechsler, 1997)

Three working memory tasks were used: digit span forwards (repeating back series of numbers), digit span backwards (repeating back series of numbers in reverse), and letter-number sequencing (sorting and recalling a series of letters and numbers). The demands on working memory increase across each of these three tasks. These are the most commonly used working memory tasks in schizophrenia research (Nuechterlein et al. 2004).

\section{Wechsler Abbreviated Scale of Intelligence (WASI)}

The WASI (Wechsler, 1999) is a standardized short and reliable measure of intelligence. The vocabulary and matrix reasoning subtests were used to obtain an estimate of IQ.

\section{IUS}

The IUS (Freeston et al. 1994) has 27 items each rated on a 1 to 5 scale. Higher scores indicate greater levels of intolerance of uncertainty. There are four subscales derived from factor analysis (Berenbaum et al. 2008): desire for predictability (e.g. 'Unforeseen events upset me greatly'); a tendency to become paralysed in the face of uncertainty (e.g. 'When I am uncertain I can't function very well'); distress in the face of uncertainty (e.g. 'The ambiguities in life stress me'); and inflexible uncertainty beliefs (e.g. 'Being uncertain means that a person is disorganized'). These subscales in the present study had high levels of internal reliability: desire for predictability, Cronbach's $\alpha=0.85$; uncertainty paralysis, Cronbach's $\alpha=0.85$; uncertainty distress, Cronbach's $\alpha=0.80$; inflexible certainty beliefs, Cronbach's $\alpha=0.76$.

\section{PSYRATS-delusions}

The PSYRATS-delusions (Haddock et al. 1999) is a sixitem multidimensional measure. It assesses the conviction, preoccupation, distress and disruption associated with delusions. Symptoms over the last week are rated. Higher scores indicate greater severity. The two assessors in the current study had high inter-rater reliability $(n=20$, intra-class correlation coefficient $=0.99)$.

\section{Positive and Negative Syndrome Scale (PANSS)}

The PANSS (Kay, 1991) is a 30-item rating instrument developed for the assessment of patients with schizophrenia. Symptoms over the last week were rated (i.e. currently present). Higher scores indicate the 
greater presence of psychiatric symptoms. The two assessors in the current study had high inter-rater reliability $(n=20$, PANSS total intra-class correlation coefficient $=0.91$ ).

\section{Green et al. Paranoid Thoughts Scale (GPTS)}

The GPTS (Green et al. 2008) is a 32-item measure of paranoid thinking. Part A assesses ideas of reference (e.g. 'It was hard to stop thinking about people talking about me behind my back') and part B assesses ideas of persecution (e.g. 'I was convinced there was a conspiracy against me'). Each item is rated on a five-point scale. Higher scores indicate greater levels of paranoid thinking. The scale was completed for the period of the previous fortnight. The internal reliability of the scale was high (Cronbach's $\alpha$ for part A was 0.90, and for part B it was 0.94).

\section{PSWQ}

The PSWQ (Meyer et al. 1990) is the most established measure of trait worry style and has been used in nonclinical and clinical populations (for a review, see Startup \& Erickson, 2006). Each of the 16 items is rated on a five-point scale. Higher scores indicate a greater tendency to worry. In the current study Cronbach's $\alpha$ for the scale was 0.79 .

\section{Perseverative Thinking Questionnaire (PTQ)}

The PTQ (Ehring et al. 2011) is a 15-item questionnaire asking about how a person typically thinks about negative problems (e.g. 'The same thoughts keep going through my mind again and again'), with each item assessed on a 0 to 4 scale. Higher scores indicate greater levels of repetitive negative thinking. Cronbach's $\alpha$ for the scale was 0.92 .

\section{Analysis}

Analyses were carried out using SPSS (version 20.0, release 20.0.0; IBM, USA). The patient group was divided into two: those showing JTC and those who did not. To test the two primary hypotheses the groups were then compared using $t$ tests on the measures of working memory and intolerance of uncertainty. Binary logistic regressions with JTC as the dependent variable $(0=$ no JTC, $1=$ JTC $)$ were then carried out to allow for analyses with covariates. All hypothesis testing was two-tailed.

\section{Results}

\section{Demographics}

The demographic details of the participants are summarized in Table 1. Levels of persecutory delusion
Table 1. Basic demographic and clinical information

\begin{tabular}{|c|c|}
\hline & $\begin{array}{l}\text { Persecutory delusions } \\
\text { group }(n=123)\end{array}$ \\
\hline Mean age, years (s.D.) & $40.6(11.2)$ \\
\hline \multicolumn{2}{|l|}{ Sex, $n$} \\
\hline Male & 73 \\
\hline Female & 50 \\
\hline \multicolumn{2}{|l|}{ Ethnicity, $n$} \\
\hline White & 113 \\
\hline Black Caribbean & 0 \\
\hline Black African & 0 \\
\hline Black other & 0 \\
\hline Indian & 3 \\
\hline Pakistani & 0 \\
\hline Chinese & 0 \\
\hline Other & 7 \\
\hline \multicolumn{2}{|l|}{ Diagnosis, $n$} \\
\hline Schizophrenia & 87 \\
\hline Schizo-affective disorder & 10 \\
\hline Delusional disorder & 10 \\
\hline Psychosis NOS & 16 \\
\hline \multicolumn{2}{|l|}{$\begin{array}{l}\text { Neuroleptic medication } \\
\text { (chlorpromazine equivalents), } n\end{array}$} \\
\hline None & 7 \\
\hline Low $(1-200 \mathrm{mg})$ & 37 \\
\hline Medium (201-400 mg) & 27 \\
\hline High (>400 mg) & 51 \\
\hline Mean PSYRATS-delusions score (S.D.) & $18.3(3.1)$ \\
\hline Mean PANSS total score (S.D.) & $79.8(13.5)$ \\
\hline Mean GPTS - part A score (S.D.) & $51.2(13.7)$ \\
\hline Mean GPTS - part B score (s.D.) & $51.0(13.9)$ \\
\hline Mean total GPTS score (S.D.) & $102.1(25.2)$ \\
\hline Mean IQ score (S.D.) & $99.4(18.4)$ \\
\hline
\end{tabular}

S.D., Standard deviation; NOS, not otherwise specified; PSYRATS, Psychotic Symptom Rating Scales; PANSS, Positive and Negative Syndrome Scale; GPTS, Green et al. Paranoid Thoughts Scale; IQ, intelligence quotient.

were high as assessed using the PSYRATS and the GPTS, with scores similar to those in other samples selected for current persecutory delusions (e.g. Foster et al. 2010). Hallucinations were common, with 75 of the patients scoring four or above on the PANSS hallucination item. Only seven patients scored four or above on the PANSS grandiosity item.

\section{JTC}

The mean number of beads drawn on the $60: 40$ task was 7.6 (S.D. =5.9). This is comparable with that found in the study of Garety et al. (2013) who reported a mean of 7.2 (S.D.=5.7) $(n=126)$. In the current study 30 patients $(24.4 \%)$ with persecutory delusions jumped 
Table 2. Neuropsychological functioning and JTC

\begin{tabular}{lllll}
\hline & JTC: mean (s.D.) & No JTC: mean (s.D.) & Mean difference (95\% CI) & $p$ \\
\hline Forward digit span & $8.9(1.8)$ & $9.8(2.5)$ & $0.9(0.1-1.8)$ & 0.033 \\
Backward digit span & $5.0(2.1)$ & $6.2(2.1)$ & $1.2(0.3-2.0)$ & 0.013 \\
Letter-number sequencing & $6.8(3.5)$ & $8.9(3.1)$ & $2.1(0.7-3.5)$ & 0.003 \\
IQ & $91.4(16.8)$ & $102.0(18.3)$ & $10.6(3.2-18.1)$ & 0.006 \\
\hline
\end{tabular}

JTC, Jumping to conclusions; S.D., standard deviation; CI, confidence interval; IQ, intelligence quotient.

to conclusions. Again, this rate of JTC is comparable with that found in the study of Garety et al. (2013) (27\% showing JTC), but it is lower than that reported by So et al. (2012) (40\%) $(n=184)$. There were no differences between those who jumped to conclusions and those who did not in levels of paranoia (GPTS total; $\left.t_{120}=0.642, p=0.522\right)$, severity of the persecutory delusions (PSYRATS total; $t_{121}=0.018, p=0.986$ ), the presence of hallucinations (PANSS hallucinations item; $t_{121}=-0.263, p=0.793$ ) or in overall psychopathology (PANSS total; $\left.t_{121}=-1.028, p=0.306\right)$. However, the patients who showed JTC did have higher levels of negative symptoms (PANSS negative; $t_{121}=-2.227$, $p=0.028)$.

\section{Neuropsychological functioning and JTC}

Those patients who jumped to conclusions, compared with those who did not, showed poorer working memory performance and had lower IQ scores (see Table 2). IQ scores correlated with forward digit span $(r=0.36, p<0.001)$, backward digit span $(r=0.50$, $p<0.001)$ and letter-number sequencing $(r=0.55$, $p<0.001)$. Letter-numbering sequencing showed the strongest association with JTC. In a binary logistic regression predicting JTC, when letter-number sequencing and IQ were entered together as independent variables they both became non-significant predictors $(p>0.1)$. The presence of negative symptoms negatively correlated with forward digit span $(r=-0.14, p=0.129)$, backward digit span $(r=-0.24, p=0.008)$, letternumber sequencing $(r=-0.24, p=0.009)$ and IQ scores $(r=-0.37, p<0.001)$. In a binary logistic regression predicting JTC, letter-number sequencing remained a significant predictor $(p=0.020)$, but negative symptoms did not $(p=0.095)$.

\section{Intolerance of uncertainty and JTC}

The total delusion group's mean IUS score of 88.4 (s.D.=24.1) was high. For example, Buhr \& Dugas (2002) reported a mean score in a non-clinical population $(n=273)$ of 54.8 (s.D. $=17.4)$, and Norton (2005) reported a mean score in a non-clinical population $(n=540)$ of 54.9 (S.D.=18.7). Dudley et al. (2011) reported a mean score of 65.2 (S.D. $=24.6$ ) in patients seen in a first-episode psychosis service $(n=77)$. The scores of the patients with persecutory delusions are closer to those of a generalized anxiety disorder group. For example, van der Heiden et al. (2012) reported a mean IUS score of 82.3 (S.D. $=21.1$ ) in a generalized anxiety disorder group $(n=42)$. There was no difference in the current study in IUS scores between patients with and without hallucinations $\left(t_{119}=-1.037, p=0.302\right)$.

Those patients who jumped to conclusions, compared with those who did not, showed lower levels of intolerance of uncertainty, although this was only statistically significant for the presence of inflexible certainty beliefs (see Table 3). Patients with JTC also reported significantly lower levels of worry, but no differences in anxiety or repetitive negative thinking. Worry positively correlated with total IUS scores $(r=0.49, p<0.001)$. When total IUS score was entered with worry as independent variables in a binary logistic regression, there was a trend for worry scores to predict JTC $(B=-0.055$, odds ratio (OR) 0.95, $p=0.067)$, but IUS scores did not predict JTC $(B=-0.008$, OR $0.99, p=0.450)$. A similar pattern was found for the IUS inflexible certainty beliefs score. In a binary logistic regression predicting JTC, there were indications that worry was a predictor $(B=-0.051$, OR 0.95, $p=0.078)$, but the IUS certainty beliefs did not predict JTC $(B=-0.073$, OR 0.93, $p=0.213$ ).

\section{Working memory, worry and JTC}

There were no significant correlations between worry and the working memory tasks (all $p>0.1$ ). In a binary logistic regression with JTC as the dependent variable, and letter-number sequencing and worry as the independent variables, both poorer working memory $(B=-0.229$, OR 0.80, $p=0.002)$ and lower levels of worry $(B=-0.082$, OR 0.922, $p=0.005)$ predicted JTC. Similarly, in a binary logistic regression, JTC was predicted by both lower IQ $(B=-0.037$, OR 0.963 , 
Table 3. Intolerance of uncertainty and JTC

\begin{tabular}{lllll}
\hline & JTC: mean (S.D.) & No JTC: mean (s.D.) & Mean difference (95\% CI) & $p$ \\
\hline IUS total & $81.6(22.2)$ & $90.6(24.4)$ & $9.1(-0.9$ to 19.0$)$ & 0.074 \\
$\quad$ Desire for predictability & $21.5(23.9)$ & $23.9(6.9)$ & $2.4(-0.4$ to 5.3$)$ & 0.088 \\
$\quad$ Uncertainty paralysis & $18.4(5.6)$ & $20.2(6.0)$ & $1.8(-0.7$ to 4.2$)$ & 0.159 \\
$\quad$ Uncertainty distress & $16.5(4.5)$ & $17.3(5.0)$ & $0.8(-1.3$ to 2.8$)$ & 0.453 \\
$\quad$ Inflexible certainty beliefs & $10.1(3.4)$ & $11.9(4.3)$ & $1.8(0.1$ to 3.5$)$ & 0.036 \\
Anxiety & $24.9(11.1)$ & $26.4(14.9)$ & $4.5(-3.6$ to 6.6$)$ & 0.565 \\
Worry & $60.7(8.4)$ & $64.8(7.6)$ & 0.9 to 7.4$)$ & 0.012 \\
Perseverative thoughts & $44.0(9.5)$ & $44.6(9.6)$ & & 0.757 \\
\hline
\end{tabular}

JTC, Jumping to conclusions; S.D., standard deviation; CI, confidence interval; IUS, Intolerance of Uncertainty Scale.

$p=0.004)$ and lower levels of worry $(B=-0.076$, OR 0.927, $p=0.007)$. When letter-number sequencing, IQ and worry were simultaneously entered as independent variables, JTC was predicted by worry $(B=-0.083$, OR 0.921, $p=0.005)$, but not significantly by working memory $(B=-0.160$, OR $0.853, p=0.070)$ or IQ $(B=-0.022$, OR $0.978, p=0.149)$.

\section{Discussion}

Two potential contributors to the JTC reasoning style were examined within a large group of patients with current persecutory delusions. JTC was considered in relation to basic information-processing capacity problems and motivational beliefs concerning uncertainty. It is the former contributor for which the most confident conclusions can be drawn. Given the similar findings of the large studies of Garety et al. (2013) and Falcone (2013), we can now be confident of an association of JTC with working memory. Arguably, studies such as that of Ormrod et al. (2012) failed to find such an association because of a small sample size. The working memory impairment was associated with levels of current intellectual functioning (and these were not separable predictors of JTC). Keeping alternative explanations in mind, gathering data, and weighing the evidence all have plausible memory load; pursuing one explanation, gathering confirmatory evidence, and reaching a definitive conclusion are likely to reduce demands on memory. Working memory difficulties via the reasoning strategies that they provoke are likely to be a factor in the persistence of delusional beliefs.

Levels of intolerance of uncertainty in the patients with persecutory delusions were high, consistent with the presence of worry in this group at a level comparable with patients with anxiety disorders. A number of studies have now identified high levels of worry in patients with persecutory delusions (e.g. Freeman \& Garety, 1999; Startup et al. 2007;
Bassett et al. 2009; Freeman et al. 2010). Against prediction, however, IUS scores were slightly higher in the patients with delusions who did not jump to conclusions compared with the patients who did jump to conclusions. Greater intolerance of uncertainty was clearly not associated with JTC, consistent with the study of Dudley et al. (2011). As originally proposed by Freeston et al. (1994), intolerance of uncertainty may, at least within a clinical psychosis sample, lead to greater information search before certainty. However, the levels of worry in the group may well explain the association. Caution is needed about over-interpreting this result before replication. This is especially the case since: there was no association with the occurrence of general repetitive negative thinking; an experimental study with patients with persecutory delusions showed no change in JTC in the period after a bout of worry (Freeman et al. 2013); and there is little evidence that anxiety disorder groups seek more data during the beads task relative to non-clinical controls (e.g. Jacobsen et al. 2012). The study provides little evidence that one form of motivational belief-intolerance of uncertainty -is a factor in JTC.

The key weakness of the study design is that it only identifies factors associated with JTC. An unmeasured variable may better explain the occurrence of JTC. Testing of causal roles is needed, for example, by manipulating working memory performance during data gathering. The group had persistent persecutory delusions, and therefore testing of different delusion types and stages of illness could result in different results. Relationships with other symptom types remain to be established; for example, in the current study there was an association of JTC with negative symptoms, but this was not found in the study of Garety et al. (2013). There are also other factors that could moderate the extent of data gathering, for example, a reliance on experiential reasoning (Freeman et al. 2012b). As the work is taken forward there will 
ideally be investigation of data gathering directly in relation to delusional ideas, and hence study of particular reasoning styles that can enhance recovery from persecutory delusions. A greater understanding of the reasoning processes that lead to erroneous beliefs becoming corrected is a clear priority for the development of a new generation of interventions for delusions.

\section{Acknowledgements}

The study was supported by a grant (no. 09/160/06) from the Efficacy and Mechanism Evaluation (EME) Programme, which is funded by the UK Medical Research Council and the UK NHS National Institute for Health Research. D.F. is supported by a UK Medical Research Council Senior Clinical Fellowship.

\section{Declaration of Interest}

None.

\section{References}

Bassett M, Sperlinger D, Freeman D (2009). Fear of madness and persecutory delusions. Psychosis 1, 39-50.

Berenbaum H, Bredemeier K, Thompson R (2008). Intolerance of uncertainty: exploring its dimensionality and associations with need for cognitive closure, psychopathology, and personality. Journal of Anxiety Disorders 22, 117-125.

Broome M, Johns L, Valli I, Woolley J, Tabraham P, Brett C, Valmaggia L, Peters E, Garety P, McGuire P (2007). Delusion formation and reasoning biases in those at clinical high risk for psychosis. British Journal of Psychiatry 191, s38-s42.

Buhr K, Dugas M (2002). The Intolerance of Uncertainty Scale: psychometric properties of the English version. Behaviour Research and Therapy 40, 931-945.

Dudley R, Daley K, Nicholson M, Shaftoe D, Spencer H, Cavanagh K, Freeston M (2013). Jumping to conclusions in first-episode psychosis: a longitudinal study. British Journal of Clinical Psychology 52, 380-393.

Dudley R, John C, Young A, Over D (1997). Normal and abnormal reasoning in people with delusions. British Journal of Clinical Psychology 36, 243-258.

Dudley R, Shaftoe D, Cavanagh K, Spencer H, Ormrod J, Turkington D, Freeston M (2011). 'Jumping to conclusions' in first-episode psychosis. Early Intervention in Psychiatry 5, 50-56.

Dugas M, Gagnon F, Ladouceur R, Freeston M (1998). Generalised anxiety disorder: a preliminary test of a conceptual model. Behaviour Research and Therapy 36, 215-226.

Ehring T, Zetsche U, Weidacker K, Wahl K, Schönfeld S, Ehlers A (2011). The Perseverative Thinking Questionnaire
(PTQ): validation of a content-independent measure of repetitive negative thinking. Journal of Behaviour Therapy and Experimental Psychiatry 42, 225-232.

Falcone A (2013). Neuropsychological and symptom correlates of the jumping to conclusions reasoning bias. PhD thesis. King's College London: London.

Foster C, Startup H, Potts L, Freeman D (2010). A randomised controlled trial of a worry intervention for individuals with persistent persecutory delusions. Journal of Behavior Therapy and Experimental Psychiatry 41, 45-51.

Freeman D, Dunn G, Startup H, Kingdon D (2012a). The effects of reducing worry in patients with persecutory delusions: study protocol for a randomized controlled trial. Trials 13, 223.

Freeman D, Evans N, Lister R (2012b). Gut feelings, deliberative thought, and paranoid ideation. Psychiatry Research 197, 119-122.

Freeman D, Garety PA (1999). Worry, worry processes and dimensions of delusions. Behavioural and Cognitive Psychotherapy 27, 47-62.

Freeman D, Garety PA (2000). Comments on the content of persecutory delusions: does the definition need clarification? British Journal of Clinical Psychology 39, 407-414.

Freeman D, Garety PA, Kuipers E, Colbert S, Jolley S, Fowler D, Dunn G, Bebbington PE (2006). Delusions and decision-making style: use of the Need for Closure Scale. Behaviour Research and Therapy 44, 1147-1158.

Freeman D, Pugh K, Vorontsova N, Antley A, Slater M (2010). Testing the continuum of delusional beliefs: an experimental study using virtual reality. Journal of Abnormal Psychology 119, 83-92.

Freeman D, Startup H, Dunn G, Černis E, Wingham G, Pugh K, Cordwell J, Kingdon D (2013). The interaction of affective with psychotic processes: a test of the effects of worrying on working memory, jumping to conclusions, and anomalies of experience in patients with persecutory delusions. Journal of Psychiatric Research 47, 1837-1842.

Freeston M, Rhéaume J, Letarte H, Dugas M, Ladouceur R (1994). Why do people worry? Personality and Individual Differences 17, 791-802.

Garety P, Freeman D (1999). Cognitive approaches to delusions: a critical review of theories and evidence. British Journal of Clinical Psychology 38, 113-154.

Garety P, Freeman D (2013). The past and future of delusion research: from the inexplicable to the treatable. British Journal of Psychiatry 203, 327-333.

Garety PA, Freeman D, Jolley S, Dunn G, Bebbington PE, Fowler D, Kuipers E, Dudley R (2005). Reasoning, emotions and delusional conviction in psychosis. Journal of Abnormal Psychology 114, 373-384.

Garety P, Joyce E, Jolley S, Emsley R, Waller H, Kuipers E, Bebbington P, Fowler D, Dunn G, Freeman D (2013).

Neuropsychological functioning and jumping to conclusions in delusions. Schizophrenia Research 150, 570-574.

Garety P, Hemsley D, Wessely S (1991). Reasoning in deluded schizophrenic and paranoid patients: biases in 
performance on a probabilistic inference task. Journal of Nervous and Mental Disease 179, 194-201.

Green C, Freeman D, Kuipers E, Bebbington P, Fowler D, Dunn G, Garety P (2008). Measuring ideas of persecution and social reference. Psychological Medicine 38, 101-111.

Haddock G, McCarron J, Tarrier N, Faragher FB (1999). Scales to measure dimensions of hallucinations and delusions: the Psychotic Symptom Rating Scales (PSYRATS). Psychological Medicine 29, 879-889.

Jacobsen P, Freeman D, Salkovskis P (2012). Reasoning bias and belief conviction in obsessive-compulsive disorder and delusions. British Journal of Clinical Psychology 51, 84-99.

Kay SR (1991). Positive and Negative Syndromes in Schizophrenia. Brunner: New York.

Kruglanski A, Webster D (1996). Motivated closing of the mind. Psychological Review 103, 263-283.

Lincoln T, Ziegler M, Mehl S, Rief W (2010). The jumping to conclusions bias in delusions. Journal of Abnormal Psychology 119, 40-49.

Menon M, Mizrahi R, Kapur S (2008). Jumping to conclusions and delusions in psychosis: relationship and response to treatment. Schizophrenia Research 98, 225-231.

Menon M, Pomarol-Clotet E, McKenna P, McCarthy R (2006). Probabilistic reasoning in schizophrenia: a comparison of the performance of deluded and nondeluded schizophrenic patients and exploration of possible cognitive underpinnings. Cognitive Neuropsychiatry 11, 521-536.

Meyer TJ, Miller ML, Metzger RL, Borkovec TD (1990). Development and validation of the Penn State Worry Questionnaire. Behaviour Research and Therapy 28, 487-495.

Moritz S, Vitzthum F, Randjbar S, Veckenstedt R, Woodward TS (2010). Detecting and defusing cognitive traps: metacognitive intervention in schizophrenia. Current Opinion in Psychiatry 23, 561-569.

Norton P (2005). A psychometric analysis of the Intolerance of Uncertainty Scale among four racial groups. Journal of Anxiety Disorders 19, 699-707.

Nuechterlein K, Barch D, Gold J, Goldberg T, Green M, Heaton R (2004). Identification of separable cognitive factors in schizophrenia. Schizophrenia Research 72, 29-39.

Ormrod J, Shaftoe D, Cavanagh K, Freeston M, Turkington D, Price J, Dudley R (2012). A pilot study exploring the contribution of working memory to "jumping to conclusions" in people with first episode psychosis. Cognitive Neuropsychiatry 17, 97-114.
So S, Freeman D, Dunn G, Kapur S, Kuipers E, Bebbington P, Fowler D, Garety P (2012). Jumping to conclusions, a lack of belief flexibility and delusional conviction in psychosis. Journal of Abnormal Psychology 121, 129-139.

Startup HM, Erickson TM (2006). The Penn State Worry Questionnaire (PSWQ). In Worry and its Psychological Disorders (ed. G. C. L. Davey and A. Wells), pp. 101-120. Wiley: Chichester.

Startup H, Freeman D, Garety PA (2007). Persecutory delusions and catastrophic worry in psychosis: developing the understanding of delusion distress and persistence. Behaviour Research and Therapy 45, 523-537.

Sternheim L, Startup H, Schmidt U (2011). An experimental exploration of behavioural and cognitive-emotional aspects of intolerance of uncertainty in eating disorder patients. Journal of Anxiety Disorders 25, 806-812.

van der Heiden C, Muris P, van de Molen H (2012). Randomized controlled trial on the effectiveness of intolerance-of-uncertainty therapy for generalized anxiety disorder. Behaviour Research and Therapy 50, 100-109.

Ventura J, Hellemann G, Thames A, Koellner V, Nuechterlein K (2009). Symptoms as mediators of the relationship between neurocognition and functional outcome in schizophrenia: a meta-analysis. Schizophrenia Research 113, 189-199.

Waller H, Freeman D, Jolley S, Dunn G, Garety P (2011). Targeting reasoning biases in delusions. Journal of Behavior Therapy and Experimental Psychiatry 42, 414-421.

Warman D, Lysaker P, Martin J, Davis L, Haudenschield S (2007). Jumping to conclusions and the continuum of delusional beliefs. Behaviour Research and Therapy 45, 1255-1269.

Wechsler D (1997). Wechsler Adult Intelligence Scale, 3rd edn. The Psychological Corporation: San Antonio, TX.

Wechsler D (1999). Wechsler Abbreviated Scale of Intelligence (WASI). The Psychological Corporation: San Antonio, TX.

Wechsler D (2001). Wechsler Test of Adult Reading (WTAR). The Psychological Corporation: San Antonio, TX.

Woodward TS, Mizrahi R, Menon M, Christensen BK (2008). Correspondences between theory of mind, jumping to conclusions, neuropsychological measures and the symptoms of schizophrenia. Psychiatry Research 170, 119-123. 